\title{
Interference Analysis for Secondary Coexistence in TV White Space
}

\author{
Angela Sara Cacciapuoti, Member, IEEE, and Marcello Caleffi, Member, IEEE
}

\begin{abstract}
In this letter, the interference among mobile, heterogeneous and independently-operated secondary networks over shared TV white space is studied. Specifically, a probabilistic model for the secondary coexistence is developed, and, stemming from this, closed-form expressions of the coexistent interference probability are derived. The analytical results show that the coexistent interference is a non-linear function of the following key parameters: (i) the number of secondary networks roaming within a geographic area; (ii) the number of users belonging to each secondary network; (iii) the traffic pattern of each secondary network; (iv) the mobility pattern of each secondary network; (v) the interference range of each secondary network. The proposed analysis is crucial and preliminary for designing any coexistence protocol, since it discloses the intrinsic relationship between environmental and system parameters. Furthermore, closed-form expressions of necessary and sufficient conditions assuring that the coexistent interference is bounded by a given threshold are derived. Simulation results validate the theoretical analysis.
\end{abstract}

Index Terms-Coexistence, TV white space (TVWS), cognitive radio.

\section{INTRODUCTION}

$\mathbf{T}$ O ACCOMMODATE rapidly increasing demand for wireless broadband services, regulatory efforts are currently ongoing in many countries to enable secondary access to channels of the TV spectrum temporarily unused by the licensed users, referred to as incumbents [1]-[3]. This group of non-contiguous vacant channels is known as TV white space (TVWS). Secondary communications in TVWS are conditioned by regulators on the ability of the secondary users (SUs) to avoid harmful interference to incumbents. To this aim, the general consensus among Ofcom, the FCC, and CEPT's SE43 working group is on the adoption of geolocation databases [4], known as White Space Databases (WSDBs), for replacing the spectrums sensing functionality [5].

However, there are no legal requirements for the coexistence among heterogeneous and independently-operated secondary network (SN) over TVWS [6]. Hence, any SN satisfying the regulations for incumbent protection can freely access to the TVWS. Consequently, multiple SNs can select the same TVWS channel for their operations and may create interference to each other, leading so to a performance degradation. This issue becomes even more severe in areas with high wireless network penetration and dense population, e.g., urban areas, due to the limited number of available TVWS channels. Fig. 1 exemplifies

Manuscript received December 19, 2014; accepted December 21, 2014. Date of publication December 29, 2014; date of current version March 6, 2015. The associate editor coordinating the review of this paper and approving it for publication was Z. Z. Lei.

The authors are with the Department of Electrical Engineering and Information Technology (DIETI), University of Naples Federico II, Naples 80125, Italy (e-mail: angelasara.cacciapuoti@unina.it; marcello.caleffi@unina.it).

Digital Object Identifier 10.1109/LCOMM.2014.2386349

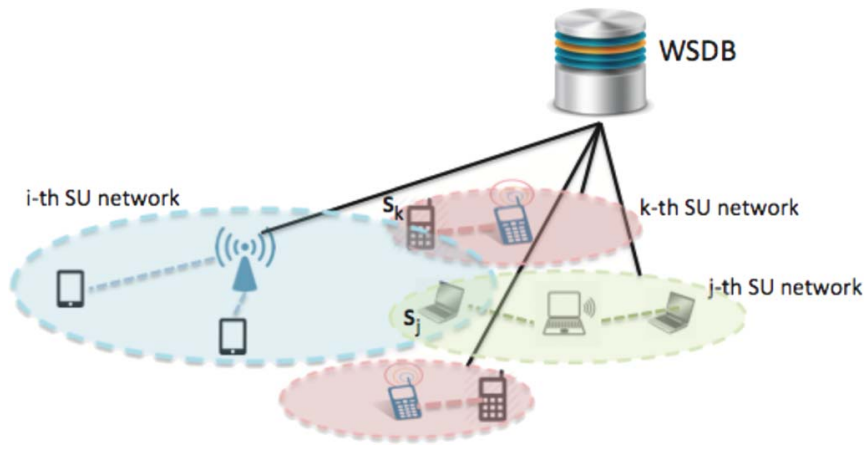

Fig. 1. Interference among Multiple Secondary Networks.

an interfering scenario where multiple heterogeneous and independently operated SNs are closely located in a certain area, and are using the same portion of the TVWS declared available by the WSDB. Specifically, the receiver $s_{j}$ of the $j$-th SN experiences interferences from the $i$-th $\mathrm{SN}$, since it is located in the overlapping portion of the coverage areas of both the SNs. A similar reasoning holds for the receiver $s_{k}$ of the $k$-th SN.

In this letter, we study the interference among mobile, heterogeneous and independently-operated SNs using the same portion of the TVWS, with the objective to determine the parameters that affect the secondary coexistence. Specifically, we develop a probabilistic model for the SU coexistence and, stemming from this, we derive closed-form expressions of the interference probability. The analytical results show that the interference among coexisting SNs is a non-linear function of the following key parameters: (i) the number of SNs roaming within a certain geographic area; (ii) the number of SUs belonging to each $\mathrm{SN}$; (iii) the traffic pattern of each $\mathrm{SN}$; (iv) the mobility pattern of each SN; (v) the interference range $^{1}$ of each $\mathrm{SN}$. The derived results disclose the intrinsic relationship between environmental and system parameters. Hence, our analysis is crucial and preliminary for designing any effective coexistence protocol. Furthermore, stemming from the developed theoretical model, we derive necessary and sufficient conditions assuring that the coexistent interference is bounded by a given threshold. Such conditions are expressed in terms of two key parameters, i.e., the interference range and the number of SUs of an arbitrary SN. Simulation results validate the theoretical derivations. To the best of our knowledge, this is the first work that reveals the non-linear dependence of the SU coexistent interference on all the aforementioned parameters.

\section{Network Model And Preliminaries}

In a geographic area $\mathbf{A}, K$ mobile, heterogeneous and independently-operated SNs use the same portion of the TVWS declared available by the WSDB. The traffic of the $i$-th SN is modeled thought the parameters $P_{i}^{\text {on }}$ and $\bar{P}_{i}^{\text {on }} \triangleq 1-P_{i}^{\text {on }}$,

\footnotetext{
${ }^{1} \mathrm{~A}$ formal definition of interference range is provided in Section II.
} 
representing the probability of an arbitrary SU belonging to the $i$-th SN being active and inactive, respectively.

Definition 1: $N_{i}$ denotes the number of SUs belonging to the $i$-th SN. The $N_{i}$ SUs move according to a general mobility model whose steady-state spatial distribution has a probability density function (pdf) denoted with $f_{\mathbf{S}_{i}}(\mathbf{s})$.

Remark 1: In the following, we assume that different SNs can move independently of each other according to different mobility models to confer generality to the analysis.

Definition 2: $M_{j}$ denotes the number of receivers belonging to the $j$-th SN.

Definition 3 (Coexistent Interference Range): Let $\mathbf{S}_{i}(t) \in \mathbf{A}$ be the position at time instant $t$ of an arbitrary $\mathrm{SU}$, say $s_{i}$, belonging to the $i$-th $\mathbf{S N}$, and let $\mathbf{S}_{j}(t) \in \mathbf{A}$ be the position at time $t$ of an arbitrary receiver, say $s_{j}$, belonging to the $j$-th SN. $s_{i}$ may interfere $s_{j}$ at time $t$ whenever they are within a distance $r_{i, j}^{\mathrm{CI}}$ referred to as coexistent interference range, i.e., $D_{i, j}(t) \triangleq\left\|\mathbf{S}_{i}(t)-\mathbf{S}_{j}(t)\right\| \leq r_{i, j}^{\mathrm{CI}}$.

Remark 2: The value of $r_{i, j}^{\mathrm{CI}}$ mainly depends on the $s_{i}$ transmission power, the $s_{j}$ sensitivity, and the adopted channel model [7]. Hence, the widely-adopted geometric model [7], [8] in Def. 3 allows us to account for the aforementioned system/environmental parameters.

Definition 4: $I_{i, j}^{\mathrm{CI}}$ denotes the event: "The $i$-th $\mathrm{SN}$ interferes the $j$-th $\mathrm{SN}$," i.e., at least one $\mathrm{SU}$ belonging to the $i$-th $\mathrm{SN}$ is active and it is within the coexistent interference range of at least one receiver belonging to the $j$-th $\mathrm{SN}$.

Definition 5 (Coexistent Interference): $I_{j}^{\mathrm{CI}}$ denotes the event: "The $j$-th SN is interfered by at least one of the other $K-1 \mathrm{SNs}$ coexisting in the same portion of the TVWS."

Definition 6 (Coexistent Interference Constraint): The Coexistent Interference Constraint $\gamma_{j}^{\mathrm{CI}}$ denotes the maximum value of the coexistence interference probability $P\left(I_{j}^{\mathrm{CI}}\right)$ that the $j$-th $\mathrm{SN}$ tolerates, i.e., $P\left(I_{j}^{\mathrm{CI}}\right) \leq \gamma_{j}^{\mathrm{CI}}$.

\section{Coexistent Interference AnAlysis}

To derive the coexistent interference probability $P\left(I_{j}^{\mathrm{CI}}\right)$, the preliminary results in Lemma 1 and Proposition 1 are required.

Lemma 1: Let $s_{i}$ be an arbitrary SU of the $i$-th SN and $s_{j}$ be an arbitrary SU of the $j$-th SN. They move according to two arbitrary mobility models in the area $\mathbf{A}$ assumed, without loss of generality, either as a line or as a square. The probability $P\left(L_{s_{i}, s_{j}}^{\mathrm{CI}}\right)$ that, at the arbitrary time instant $t, s_{i}$ and $s_{j}$ are within the coexistent interference range $r_{i, j}^{\mathrm{CI}}$ is given by:

$$
P\left(L_{S_{i}, s_{j}}^{\mathrm{CI}}\right)= \begin{cases}r_{i, j}^{\mathrm{CI}} 2\left(f_{S_{i}} \otimes f_{S_{j}}\right)(v) u(v) d v, & A=[0, a] \\ 0 & \\ r_{i, j}^{\mathrm{CI}} & \\ \int_{0}^{\mathrm{CI}} 4\left(f_{S_{i}} \otimes f_{S_{j}}\right)(v) \otimes & \\ \left.\left(f_{S_{i}} \otimes f_{S_{j}}\right)(v)\right] u(v) d v, & \mathbf{A}=[0, a]^{2}\end{cases}
$$

where $u(\cdot)$ denotes the step function, and $\left(f_{S_{i}} \otimes f_{S_{j}}\right)(\cdot)$ denotes the convolution between the pdfs of the one-dimensional steady-state spatial distributions of the SUs belonging to the $i$-th and $j$-th SNs, when they move within a line, i.e., $A=[0, a]$.

Proof: By accounting for Def. 3, it results $P\left(L_{s_{i}, s_{j}}^{\mathrm{CI}}\right) \triangleq$ $P\left(D_{i, j}(t) \leq r_{i, j}^{\mathrm{CI}}\right)=\int_{0}^{r_{i, j}^{\mathrm{CI}}} f_{D_{i, j}}(v) d v$, with $f_{D_{i, j}}(\cdot)$ the pdf of $D_{i, j}$.
For lines $A=[0, a]$, by using the Random Variable Transformation Theorem [9], and by reasoning similarly to [10], $f_{D_{i, j}}(\cdot)$ is equal to $2\left(f_{S_{i}} \otimes f_{S_{j}}\right)(\mathrm{v}) u(\mathrm{v})$. For squared areas $\mathbf{A}=$ $[0, a] \times[0, a] \triangleq[0, a]^{2}$, by using the independence and identical distribution of the random variables representing the distances in each dimension, $f_{D_{i, j}}(\cdot)$ is the convolution of the pdf of $D_{i, j}$ within a line. Hence, by using the result obtained for the onedimensional case, the proof follows.

Remark 3: $P\left(L_{s_{i}, s_{j}}^{\mathrm{CI}}\right)$ does not depend on $t$ since the SUs are moving according to their steady-state spatial distributions.

Proposition 1: The $j$-th $\mathrm{SN}$ is interfered by the $i$-th $\mathrm{SN}$ with a probability $P\left(I_{i, j}^{\mathrm{CI}}\right)$ given by:

$$
\begin{aligned}
P\left(I_{i, j}^{\mathrm{CI}}\right)= & \sum_{n=1}^{N_{i}}\left(\begin{array}{c}
N_{i} \\
n
\end{array}\right)\left[P_{i}^{\mathrm{on}}\right]^{n}\left[\bar{P}_{i}^{\mathrm{on}}\right]^{N_{i}-n} \sum_{v=1}^{n}\left(\begin{array}{l}
n \\
v
\end{array}\right) \\
& \times\left[1-\bar{P}\left(L_{s_{i}, s_{j}}^{\mathrm{CI}}\right)^{M_{j}}\right]^{v} \bar{P}\left(L_{s_{i}, s_{j}}^{\mathrm{CI}}\right)^{M_{j}(n-v)}
\end{aligned}
$$

where $\bar{P}\left(L_{s_{i}, s_{j}}^{\mathrm{CI}}\right) \triangleq 1-P\left(L_{s_{i}, s_{j}}^{\mathrm{CI}}\right)$ is derived in Lemma 1.

Proof: To prove the proposition, we first need to evaluate the probability $P\left(L_{s_{i}, j}^{\mathrm{CI}}\right)$ of an arbitrary $s_{i}$ of the $i$-th $\mathrm{SN}$ to be within the coexistent interference range $r_{i, j}^{\mathrm{CI}}$ of at least one receiver $s_{j}$ of the $j$-th $\mathrm{SN}$. By exploiting the independence of the SU movements, the identical steady-state spatial distribution of the SUs belonging to the same SN and Def. 3, it results $P\left(L_{s_{i}, j}^{\mathrm{CI}}\right)=1-\prod_{l=1}^{M_{j}}\left[1-P\left(L_{s_{i}, s_{l}}^{\mathrm{CI}}\right)\right]=1-\bar{P}\left(L_{s_{i}, s_{j}}^{\mathrm{CI}}\right)^{M_{j}}$, with $\bar{P}\left(L_{s_{i}, s_{j}}^{\mathrm{CI}}\right) \triangleq 1-P\left(L_{s_{i}, s_{j}}^{\mathrm{CI}}\right)$ given in (1). Now we can evaluate $P\left(I_{i, j}^{\mathrm{CI}}\right)$. By exploiting Def. 4 and the independence of the traffic and mobility patterns, one has $P\left(I_{i, j}^{\mathrm{CI}}\right)=$ $\sum_{n=1}^{N_{i}}\left(\begin{array}{c}N_{i} \\ n\end{array}\right)\left(P_{i}^{\text {on }}\right)^{n}\left(\bar{P}_{i}^{\text {on }}\right)^{N_{i}-n} \sum_{v=1}^{n}\left(\begin{array}{c}n \\ v\end{array}\right) P\left(L_{s_{i}, j}^{\mathrm{CI}}\right)^{v} \bar{P}\left(L_{s_{i}, j}^{\mathrm{Cl}}\right)^{n-v} \quad$ with $\bar{P}\left(L_{s_{i}, j}^{\mathrm{CI}}\right) \triangleq 1-P\left(L_{s_{i}, j}^{\mathrm{CI}}\right)$. By substituting the expression of $P\left(L_{s_{i}, j}^{\mathrm{CI}}\right)$ in the above equation, the proof follows.

Remark 4: In Proposition 1, we assume the SUs belonging to the same SN being identical distributed, since this is very reasonable and widely adopted in literature. Nevertheless, this assumption can be removed and, in such a case, $P\left(I_{i, j}^{\mathrm{CI}}\right)$ can be obtained by using $P\left(L_{s_{i}, j}^{\mathrm{CI}}\right)=1-\prod_{l=1}^{M_{j}} \bar{P}\left(L_{S_{i}, S_{l}}^{\mathrm{CI}}\right)$.

Theorem 1: The $j$-th $\mathrm{SN}$ is interfered by at least one of the other $K-1 \mathrm{SNs}$ with a probability equal to:

$$
P\left(I_{j}^{\mathrm{CI}}\right)=1-\prod_{\substack{l=1 \\ l \neq j}}^{K}\left[\bar{P}_{l}^{\mathrm{on}}+P_{l}^{\mathrm{on}} \bar{P}\left(L_{s_{l}, s_{j}}^{\mathrm{CI}}\right)^{M_{j}}\right]^{N_{l}}
$$

where $P\left(I_{l, j}^{\mathrm{CI}}\right)$ is given in Proposition 1 .

Proof: According to Def. 5, it results $P\left(I_{j}^{\mathrm{CI}}\right)=1-$ $\prod_{\substack{l=1 \\ l \neq j}}^{K}\left[1-P\left(I_{l, j}^{\mathrm{C}}\right)\right]$. By substituting (2) in the above equation and by exploiting the Binomial Theorem [11], one has: $P\left(I_{j}^{\mathrm{CI}}\right)=$ $1-\prod_{\substack{l=1 \\ l \neq j}}^{K}\left\{1-\sum_{n=1}^{N_{l}}\left(\begin{array}{c}N_{l} \\ n\end{array}\right)\left(P_{l}^{\mathrm{on}}\right)^{n}\left(\bar{P}_{l}^{\mathrm{on}}\right)^{N_{l}-n}\left[1-\bar{P}\left(L_{s_{l}, s_{j}}^{\mathrm{Cl}}\right)^{n M_{j}}\right]\right\}$. By using the notable equality $\sum_{n=1}^{N}\left(\begin{array}{l}N \\ n\end{array}\right) p^{n}(1-a)^{K n}(1-p)^{N-n}=$ $(1-p)^{N}\left(\left(\left(-p(1-a)^{K}+p-1\right) /(p-1)\right)^{N}-1\right)$ in the previous equation, (3) follows.

Remark 5: The compact and elegant closed-form expression of $P\left(I_{j}^{\mathrm{CI}}\right)$, derived In Theorem 1, allows us to disclose:

i) all the parameters affecting the coexistent interference, i.e., the number $K$ of SNs roaming within the area $\mathbf{A}$, the 
number $N_{l}$ of SUs belonging to the $l$-th $\mathrm{SN}$, the number $M_{j}$ of receivers belonging to the $j$-th $\mathrm{SN}$, the SU traffic patterns through $\left\{P_{\mathrm{on}, l}\right\}$, the SU mobility patterns through the pdfs $\left\{f_{\mathbf{S}_{\mathbf{k}}}\left(\mathbf{s}_{k}\right)\right\}$ of the steady-state spatial distributions and the coexistent interference ranges through $\left\{P\left(L_{s_{l}, s_{j}}^{\mathrm{CI}}\right)\right\}$;

ii) the non-linear dependence of the coexistence interference from the aforementioned key parameters.

In the following, we exploit the result derived in Theorem 1 for specializing the constraint $P\left(I_{j}^{\mathrm{CI}}\right) \leq \gamma_{j}^{\mathrm{CI}}$ in terms of two key parameters, i.e., $N_{i}$ and $r_{i, j}^{\mathrm{CI}}$.

Proposition 2: A necessary and sufficient condition assuring the coexistent interference constraint $P\left(I_{j}^{\mathrm{CI}}\right) \leq \gamma_{j}^{\mathrm{CI}}$ on the $j$-th $\mathrm{SN}$ is that the number $N_{i}$ of SUs belonging to the arbitrary $i$-th $\mathrm{SN}$ satisfies the following inequality:

$$
N_{i} \leq \frac{\ln \left(1-\gamma_{j}^{\mathrm{CI}}\right)-\sum_{\substack{l=1 \\ l \neq j \\ l \neq i}}^{K} N_{l} \ln \left(\bar{P}_{l}^{\mathrm{on}}+P_{l}^{\mathrm{on}} \bar{P}\left(L_{s_{l}, s_{j}}^{\mathrm{CI}}\right)^{M_{j}}\right)}{\ln \left(\bar{P}_{i}^{\mathrm{on}}+P_{i}^{\mathrm{on}} \bar{P}\left(L_{s_{i}, s_{j}}^{\mathrm{CI}}\right)^{M_{j}}\right)} .
$$

Proof: By substituting (3) in $P\left(I_{j}^{\mathrm{CI}}\right) \leq \gamma_{j}^{\mathrm{CI}}$, with algebraic manipulations, it results $\left[\bar{P}_{i}^{\text {on }}+P_{i}^{\text {on }} \bar{P}\left(L_{s_{i}, s_{j}}^{\mathrm{Cl}}\right)^{M_{j}}\right]^{N_{i}} \geq$ $\frac{1-\gamma_{j}^{\mathrm{CI}}}{\prod_{\substack{l=1 \\ l \neq j \\ l \neq j}}^{K}\left[P_{l}^{\mathrm{on}}+P_{l}^{\mathrm{on}} \bar{P}\left(L_{S_{l}, s_{j}}^{\mathrm{CI}}\right)^{M_{j}}\right]^{N_{l}}}$ when $\prod_{\substack{l=1 \\ l \neq j \\ l \neq i}}^{K}\left[1-P\left(I_{l, j}^{\mathrm{CI}}\right)\right] \neq 0$ i.e.,

$P\left(I_{l, j}^{\mathrm{C}}\right) \neq 1$ with $l \in\{1, \ldots K\}, l \neq i, l \neq j$. By applying the logarithm function with basis $\left\{\bar{P}_{i}^{\text {on }}+P_{i}^{\text {on }} \bar{P}\left(L_{s_{i}, s_{j}}^{\mathrm{CI}}\right)^{M_{j}}\right\}$ to the above inequality and by recognizing that this logarithm is a decreasing function of its argument since $\left\{\bar{P}_{i}^{\mathrm{on}}+P_{i}^{\mathrm{on}} \bar{P}\left(L_{s_{i}, s_{j}}^{\mathrm{CI}}\right)^{M_{j}}\right\} \leq 1$, the proof follows.

Corollary 1: A necessary condition assuring the coexistent interference constraint $P\left(I_{j}^{\mathrm{CI}}\right) \leq \gamma_{j}^{\mathrm{CI}}$ on the $j$-th $\mathrm{SN}$ is that the number $N_{i}$ of SUs belonging to the $i$-th SN satisfies the following inequality:

$$
N_{i} \leq \frac{\ln \left(1-\gamma_{j}^{\mathrm{CI}}\right)}{\ln \left(\bar{P}_{i}^{\mathrm{on}}+P_{i}^{\mathrm{on}} \bar{P}\left(L_{s_{i}, s_{j}}^{\mathrm{CI}}\right)^{M_{j}}\right)} .
$$

Proof: Since $P\left(I_{j}^{\mathrm{CI}}\right) \geq P\left(I_{i, j}^{\mathrm{CI}}\right)$, by exploiting the coexistent interference constraint, it results $P\left(I_{i, j}^{\mathrm{CI}}\right) \leq \gamma_{j}^{\mathrm{CI}}$. This inequality represents a necessary but not sufficient condition to assure $P\left(I_{j}^{\mathrm{CI}}\right) \leq \gamma_{j}^{\mathrm{CI}}$. By reasonings as in Prop. 2 and by substituting (2) in $P\left(I_{i, j}^{\mathrm{CI}}\right) \leq \gamma_{j}^{\mathrm{CI}}$, the proof follows.
Proposition 3: A necessary and sufficient condition assuring the coexistent interference constraint $P\left(I_{j}^{\mathrm{CI}}\right) \leq \gamma_{j}^{\mathrm{CI}}$ on the $j$-th $\mathrm{SN}$ is that the coexistent interference range $r_{i, j}^{\mathrm{CI}}$ satisfies the inequality (6), shown at the bottom of the page.

Proof: By reasoning similarly as in Prop. 2, one recognizes that $P\left(I_{j}^{\mathrm{CI}}\right) \leq \gamma_{j}^{\mathrm{CI}}$ is equivalent to: $P\left(L_{s_{i}, s_{j}}^{\mathrm{Cl}}\right) \leq 1-$

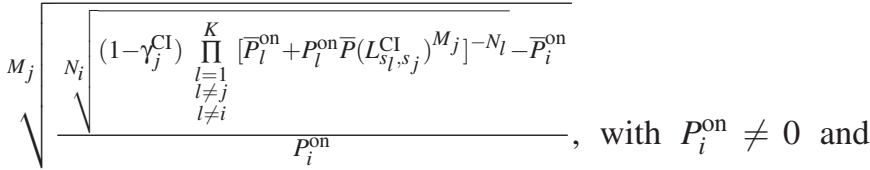
$P\left(L_{s_{i}, s_{j}}^{\mathrm{CI}}\right)$ derived in Lemma 1. If $r_{i, j}^{\mathrm{CI}}<<a$, i.e., the coexistent interference range is smaller than the dimension of the area $\mathbf{A}$, as reasonable, $P\left(L_{s_{i}, s_{j}}^{\mathrm{CI}}\right) \simeq\left\{\begin{array}{ll}\frac{2 r_{i, j}^{\mathrm{Cl}}}{a}, & A=[0, a] \\ \frac{\pi\left(r_{i, j}^{\mathrm{Cl}}\right)^{2}}{a^{2}}, & \mathbf{A}=[0, a]^{2}\end{array}\right.$. By substituting these in the derived inequality, the proof follows.

Corollary 2: A necessary condition assuring the coexistent interference constraint $P\left(I_{j}^{\mathrm{CI}}\right) \leq \gamma_{j}^{\mathrm{CI}}$ on the $j$-th $\mathrm{SN}$ is that the coexistent interference range $r_{i, j}^{\mathrm{CI}}$ satisfies the following inequality:

$$
r_{i, j}^{\mathrm{CI}} \leq \begin{cases}\frac{a}{2}\left[1-\sqrt[M_{j}]{\frac{N_{i} \sqrt{\left(1-\gamma_{j}^{\mathrm{CI}}\right)}-\bar{P}_{i}^{\mathrm{on}}}{P_{i}^{\text {On }}}}\right], & A=[0, a] \\ \sqrt[\frac{a^{2}}{\pi}\left[1-\sqrt[M_{j}]{\frac{N_{i} \sqrt{\left(1-\gamma_{j}^{\mathrm{CI}}\right)}-\bar{P}_{i}^{\text {on }}}{P_{i}^{\text {on }}}}\right]]{ }, & \mathbf{A}=[0, a]^{2} .\end{cases}
$$

Proof: The proof follows by reasonings as in Prop. 3 and by observing that, for $K>1, P\left(I_{j}^{\mathrm{CI}}\right) \geq P\left(I_{i, j}^{\mathrm{CI}}\right)$.

\section{VAlidation of the Theoretical Results}

Here, we validate the theoretical results through Monte Carlo simulations, along with real-world application examples. We consider $K=3$ SNs moving according to the Random Walk mobility model [8]. For each SN, we generate $10^{4}$ topologies by placing the users randomly in a squared region $\mathbf{A}$. Then, for each topology, we let the users move according to the adopted mobility models for enough time to reach a steady-state distribution. The simulation setting is: $r_{2,1}^{\mathrm{CI}} / a=0.01, r_{3,1}^{\mathrm{CI}} / a=$ $0.02, P_{2}^{\text {on }}=0.75$, and $P_{3}^{\text {on }}=0.5$.

Fig. 2 shows the probability $P\left(I_{1}^{\mathrm{CI}}\right)$ of the coexistent interference induced by the 2-th and 3-th SNs on the 1-th SN, as the number $M_{1}$ of receivers belonging to the 1-th SN

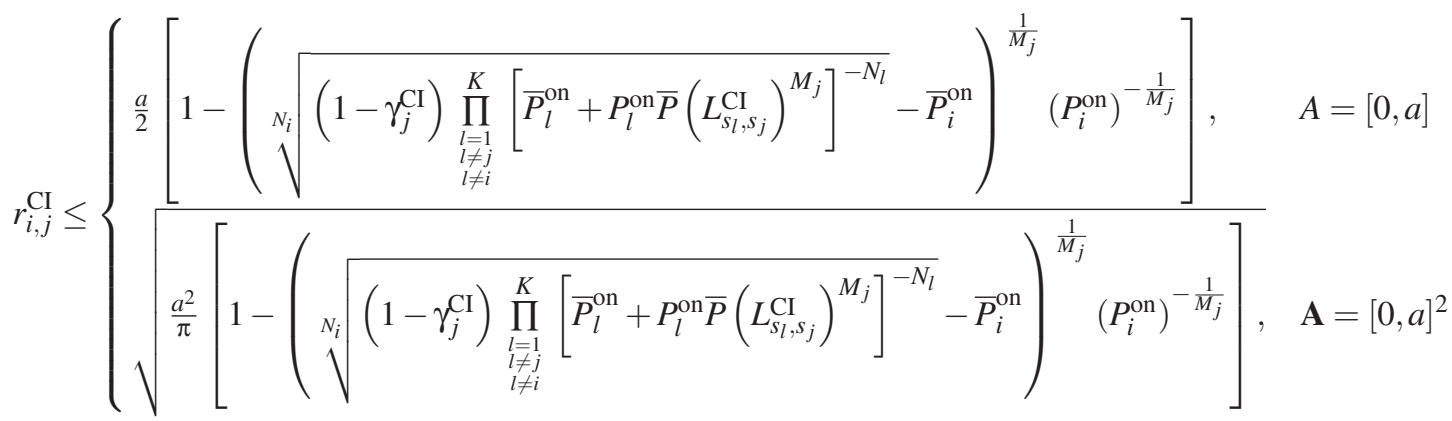




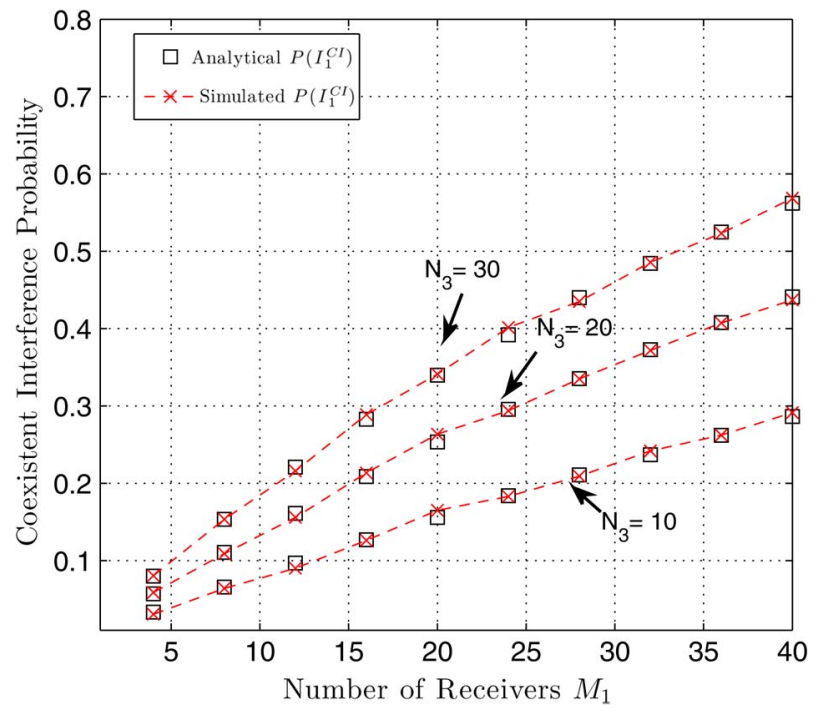

Fig. 2. $P\left(I_{j}^{\mathrm{CI}}\right)$ vs $M_{j}$.

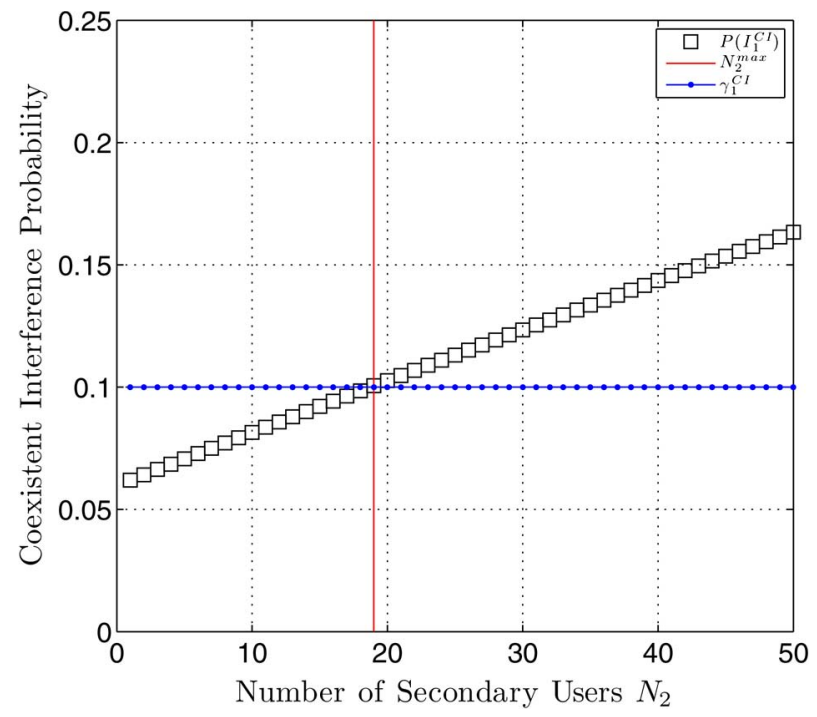

Fig. 3. Coexistent Interference Constraint: Optimal $N_{i}$.

increases, when $N_{2}=10$ and for three values of $N_{3}$. First, we note that the theoretical results (Theorem 1) matches very well the simulation results. Then, when $M_{1}$ increases, $P\left(I_{1}^{\mathrm{Cl}}\right)$ increases as well, since the probability of at least one out of $M_{1}$ receivers being inside the interference ranges of at least one SU belonging to the 2-th and/or the 3-th SNs increases. Similar comments hold when $N_{3}$ increases. Furthermore, Fig. 3 shows $P\left(I_{1}^{\mathrm{CI}}\right)$ as $N_{2}$ increases, when $N_{3}=10, M_{1}=10$ and the coexistent interference constraint $\gamma_{1}^{\mathrm{CI}}=0.1$. The results validate the theoretical analysis of Proposition 2, since when $N_{2}$ is greater than the upper bound derived in (4) and denoted in the figure with $N_{2}^{\max }, P\left(I_{1}^{\mathrm{CI}}\right)>\gamma_{1}^{\mathrm{CI}}$.

Case of Study: To further discuss the derived results, we consider the real-world application scenario described in [6], where IEEE $802.15 .4 \mathrm{~m}$ in $50 \mathrm{kbps}$ mode, IEEE 802.11 af in 6.5 Mbps mode and IEEE 802.22 in 4.54Mbps mode systems are co-located in the same TVWS band within an area $\mathbf{A}=$ $[0, a]^{2}$ with $a=1 \mathrm{Km}$. By setting the interference ranges in agreement with the experimental results reported in [6], our analysis reveals that a IEEE 802.11af network can accommodate at maximum $N_{2}^{\max }=17$ users to satisfy the interference constraint $\gamma_{1}^{\mathrm{CI}}=0.1$ on a IEEE $802.15 .4 \mathrm{~m}$ network constituted by 10 receivers, when in $\mathbf{A}$ there is one transmitter IEEE 802.22. If the side area grows to $a=2 \mathrm{Km}, N_{2}^{\max }$ is equal to 70 . Furthermore, if the interference constrained is stressed and set equal to $\gamma_{1}^{\mathrm{CI}}=0.05$ without changing the other parameters, the IEEE 802.11af network can accommodate in an area of side $a=1 \mathrm{Km}$ at maximum $N_{2}^{\max }=8$, whereas if the side area grows to $a=2 \mathrm{Km}, N_{2}^{\max }$ is equal to 33 .

\section{REFERENCES}

[1] "In the matter of unlicensed operation in the tv broadcast bands: third memorandum opinion and orderr," FCC, Washington, DC, USA, Tech. Rep. 12-36A1, Aug. 2012.

[2] "Regulatory Requirements for White Space Device in the UHF TV Band," OFCOM, Jul. 4, 2012.

[3] "Technical and operational requirements for the operation of white spaces devices under geo-location approach," CEPT, ECC, Copenhagen, Denmark, ECC Report 186, Jan. 2013.

[4] M. Caleffi and A. S. Cacciapuoti, "Database access strategy for tv white space cognitive radio networks," in Proc. 11th Annu. IEEE Int. Conf. SECON, Jun. 2014, pp. 34-38.

[5] A. Cacciapuoti, M. Caleffi, D. Izzo, and L. Paura, "Cooperative spectrum sensing techniques with temporal dispersive reporting channels," IEEE Trans. Wireless Commun., vol. 10, no. 10, pp. 3392-3402, Oct. 2011.

[6] C.-S. Sum, M.-T. Zhou, L. Lu, F. Kojima, and H. Harada, "Performance and coexistence analysis of multiple ieee $802 \mathrm{wpan} / \mathrm{wlan} / \mathrm{wran}$ systems operating in tv white space," in Proc. IEEE DYSPAN, Apr. 2014, pp. 145-148.

[7] K. Xu, M. Gerla, and S. Bae, "How effective is the ieee $802.11 \mathrm{rts} / \mathrm{cts}$ handshake in ad hoc networks," in Proc. IEEE GLOBECOM, Nov. 2002, pp. $72-76$.

[8] H. Cai and D. Y. Eun, "Crossing over the bounded domain: From exponential to power-law intermeeting time in mobile ad hoc networks," IEEE/ACM Trans. Netw., vol. 17, no. 5, pp. 1578-1591, Oct. 2009.

[9] A. Papoulis, Probability, Random Variables, Stochastic Processes. New York, NY, USA: McGraw-Hill, 1984.

[10] A. S. Cacciapuoti, I. F. Akyildiz, and L. Paura, "Primary-user mobility impact on spectrum sensing in cognitive radio networks," in Proc. IEEE 22nd Int. Symp. PIMRC, Sep. 2011, pp. 451-456.

[11] M. Abramowitz and I. Stegun, Handbook of Mathematical Functions with Formulas, Graphs, Mathematical Tables. New York, NY, USA: Dover, 1972. 